\title{
Oncolytic Properties of Ampakines In Vitro
}

\author{
DANIEL P. RADIN, RICHARD PURCELL and ARNOLD S. LIPPA
}

RespireRx Pharmaceuticals, Inc., Glen Rock, NJ, U.S.A.

\begin{abstract}
Background/Aim: The 5-year survival rate of glioblastoma (GBM) is $\sim 10 \%$, demonstrating that a new therapeutic modality for this cancer is desperately needed. Complicating the search for such a modality is that most large molecules cannot pass through the blood brain barrier, so molecules demonstrating efficacy in vitro may not be useful in vivo because they never reach the brain. Recently, the selective serotonin reuptake inhibitor (SSRI) fluoxetine (FLX) was identified as an effective agent in targeting GBM in vitro and in vivo by agonizing AMPA-glutamate receptors (AMPARs), eliciting massive calcium influx and mitochondrial calcium overload and apoptosis. Materials and Methods: In the current study, we used a colorimetric cell viability assay to determine if we could enhance the oncolytic effect of FLX in vitro by pretreating cells with an AMPAR-positive allosteric modulator (Ampakine). Results: Our results demonstrated for the first time that concentrations of the Class I ampakine CX614, which increase AMPAR agonist binding affinity, possess oncolytic activity as a sole agent and synergistically reduce GBM viability when paired with FLX. FLX also demonstrates a dosedependent induction of apoptosis in cancer cells originating outside the CNS that overexpress calcium-permeable AMPARs. Likewise, CX614 inhibits cancer cell viability in a dosedependent fashion and its combination with FLX synergistically reduces cell viability. These effects of CX614 were not seen with the Class II ampakines, CX717 and CX1739. Conclusion: CX614 inhibits the growth of multiple cancers in vitro and bolsters the oncolytic activity of FLX in several cancers.
\end{abstract}

The $\alpha$-amino-3-hydroxy-5-methyl-4-isoxazolepropionic acid (AMPA) receptors (AMPAR) are one of three ionotropic glutamate receptors in the central nervous system (CNS) (in addition to NMDA and kainate receptors) that mediate fast, excitatory neurosynaptic transmission and govern the induction of long-term potentiation, a process thought to be

Correspondence to: Daniel Radin, RespireRx Pharmaceuticals Inc., 126 Valley Road, Glen Rock, NJ 07452, U.S.A. Tel: +1 2016790671, Fax: +1 2014930887, e-mail: dradin@respirerx.com

Key Words: Ampakine, Ampa receptor, apoptosis, calcium influx, SSRI, synergy. crucial for the consolidation of learning and memory $(1,2)$. Over the past two decades, considerable strides have been made in understanding AMPAR pharmacology, kinetics and physiology by employing positive allosteric modulators (PAMs) to delineate their function (3). For convenience, the term ampakines has been used to describe various classes of drugs that act as PAM.

Ampakines are further categorized as Class I ampakines, also known as high-impact ampakines, and Class II ampakines, also known as low-impact ampakines (3). Class I or high-impact ampakines increase AMPAR agonist binding affinity by stabilizing the channel open confirmation of the AMPAR, thereby offsetting desensitization, and enhancing AMPAergic steady-state currents up to 100-fold (4). Class II ampakines are believed to increase the probability of channel opening and thereby increase current peak amplitude, but do little to desensitization kinetics and agonist binding affinity (4).

Recently, it was shown that the selective serotonin reuptake inhibitor (SSRI) fluoxetine (FLX) increases intracellular calcium levels of hippocampal neurons and astrocytes by binding to the agonist binding site of the AMPAR and inducing rapid transmembrane calcium influx (5). Furthermore, Liu et al. showed that calcium-permeable AMPAR subunits are overexpressed in several glioblastoma cell lines and FLX induced apoptosis in these cell types at micromolar concentrations by overloading the mitochondria with calcium, inducing the release of caspase co-factor cytochrome $c$ and setting off the canonical apoptotic program (5).

In the current work, we set out to determine any potential oncolytic effects of members of both classes of ampakines and whether the anti-tumor activity of FLX could be augmented by pre-treatment with ampakines.

\section{Materials and Methods}

Cell culture. U87 (Glioblastoma), Panc-1 (pancreatic adenocarcinoma) and HT29 (colorectal adenocarcinoma) were kind gifts from Mrs. Donna Leonardi (Bergen County Technical Schools, Hackensack, NJ, USA). They were chosen for this study because they express functional AMPAR receptor subunits (5-8). They were maintained in DMEM media (ATCC) and supplemented with $10 \%$ FBS (ATCC) and 1\% Penicillin/Streptomycin and maintained in an incubator at $37^{\circ} \mathrm{C}$ with $95 \%$ air and $5 \% \mathrm{CO}_{2}$. 
Reagents. Fluoxetine was purchased from TCI chemicals (Portland, Oregon, USA) and dissolved in complete DMEM media. CX614 was purchased from Tocris (Bristol, UK) and dissolved in DMSO (ATCC) as a concentrated stock solution before being added to cells. CX717 (1-(Benzofurazan-5-ylcarbonyl)morpholine) and CX1739 (N-Methyl-N-(tetrahydro-2H-pyran-4-yl)-2,1,3-benzoxadiazole-5carboxamide) were synthesized by RespireRx Pharmaceuticals (Glen Rock, NJ, USA) and dissolved in complete DMEM media.

Cell viability assay. U87, Panc-1 or HT29 cells were seeded in quadruplicate at a density of 6,000 cells/well in complete DMEM and incubated overnight. Cells were then treated with increasing concentrations of fluoxetine, CX717, CX1739 or CX614. For interaction studies, cells were pre-treated with indicated concentrations of CX1739 or CX614 for 5 min before fluoxetine administration. Cells were then incubated for a following $24 \mathrm{~h}$. Following this incubation, $20 \mu \mathrm{l}$ MTS solution (Promega, Madison, Wisconsin, USA) was added to each well and incubated for a further $2 \mathrm{~h}$. Plates were read at $490 \mathrm{nM}$ using the iMARK ELISA plate reader (Bio-Rad, Portland, Maine, USA). Data are normalized to vehicle-treated cells. Protocol was adapted from Radin et al. (9).

Yo-pro-1 apoptotic stain. After U87, Panc-1 or HT29 cells were treated with fluoxetine or CX614 for $24 \mathrm{~h}$, cells were stained with $5 \mathrm{uM}$ yo-pro-1 for $20 \mathrm{~min}$ and then visualized with the Xcite fluorescent microscope.

Data analysis. Data were analyzed using a one-way ANOVA (Statplus) and a student's $t$-test. King's Synergy (KS) formula was used to determine synergistic interactions between CX614 and fluoxetine (10). Alpha value was set at $p<0.05$.

\section{Results}

Our initial studies investigated the oncolytic effects of FLX in U87 glioblastoma cells in addition to Panc1 pancreatic cancer cells and HT29 colorectal adenocarcinoma cells. For all cell types, FLX produced a dose-dependent decline in cell viability with significant reductions at 40 and $80 \mu \mathrm{M}$ (Figure 1, $p<0.01$, $t$-test). FLX also induced apoptosis in U87 and Panc1 cells at $40 \mu \mathrm{M}$ (data not shown) and in all three cancer cell types at $80 \mu \mathrm{M}$ (data not shown). Our data are consistent with previous findings (5). Panc1's sensitivity to FLX was encouraging due to the consistently poor prognoses associated with pancreatic neoplasias underpinned with limited pharmacological and surgical interventions.

CX614, a class 1 AMPAR PAM, also produced a dose dependent decline in cell viability of all 3 cancer types tested (Figure 2, ANOVA, $p<0.001$ ) and induced a small amount of apoptosis at 1,200 and 1,600 $\mu \mathrm{M}$ doses (data not shown). CX717 and CX1739 are two Class II ampakines that do not significantly increase agonist binding affinity and do not significantly augment the steady-state current elicited by AMPAR agonists. Instead, they are believed to increase the likelihood of channel opening and only increase the peak amplitude of the AMPAergic current (4). Concentrations as high as 2,500 $\mu \mathrm{M}$ CX717 and $800 \mu \mathrm{M} \mathrm{CX1739} \mathrm{(limit} \mathrm{of}$ solubility) did not significantly alter cell viability of U87 cells (data not shown). Although this class of AMPAR PAM failed to significantly alter U87 cell viability, this finding demonstrates that novel AMPAR PAMs can be differentiated using the cell viability assay.

Finally, we examined the extent to which ampakines could augment the oncolytic effects of FLX administration. results demonstrate that in U87 cells, several combinations of CX614 and FLX synergistically reduced cell viability (Figure 3a, KS $p<0.05$ ). In Panc1 cells, $400 \mu \mathrm{M}$ CX614 significantly bolstered the oncolytic effects of FLX (Figure $3 b, \mathrm{KS} p<0.05)$. In HT29 cells, pre-treatment with $100 \mu \mathrm{M}$ or $400 \mu \mathrm{M}$ CX614 significantly bolstered FLX's oncolytic actions (Figure 3c, KS $p<0.05$ ). Also important is that CX1739, a class II ampakine failed to enhance FLX's oncolytic effects in U87 cells (data not shown).

\section{Discussion}

In the current study, CX614, a typical Class I ampakine, but not the Class II ampakines, CX717 and CX1739, was observed to possess intrinsic oncolytic activity and to enhance the oncolytic activity of FLX in U87 glioblastoma cells using concentrations that have been reported to enhance AMPAR agonist binding (11). In addition, FLX was shown to possess oncolytic activity against pancreatic and colon cancer cell lines at similar micromolar concentrations. The results with Panc-1, a commonly used pancreatic cancer cell line, were particularly encouraging because of the dismal prognoses associated with most pancreatic cancers. Novel treatments are needed to treat this type of cancer, and repurposing an FDA-approved drug such as FLX would greatly reduce the time and cost for clinical development.

Several studies have reported the oncolytic activity of FLX in several cancers, due to increased intracellular calcium levels and augmented ROS production and release by mitochondria into the cytoplasm (12). However, it was only recently that FLX was identified as an AMPAR agonist and that pretreatment of glioma cells with an AMPAR antagonist reduced the oncolytic effects of FLX (5). FLX also was shown to selectively execute glioma cells and spare healthy neurons and astrocytes because glioma cells massively over-express calcium-permeable subunits of the AMPAR. In vivo, FLX demonstrated efficacy similar to temozolomide, a standard of care for managing patients with glioblastoma (5). In addition, the present results demonstrate that FLX might possess utility as an oncolytic agent for cancers expressing CP-AMPARs regardless of tissue of origin.

On a separate note, it has been shown that acute administration of Class I ampakines increases neuronal proliferation (13) and BDNF production in organotypic cultures (14). In addition to being implicated in neuronal 


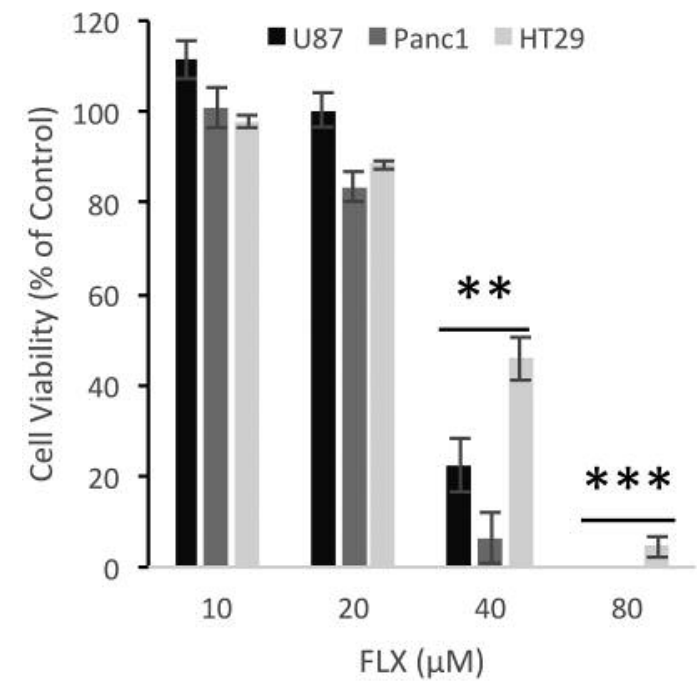

Figure 1. FLX is oncolytic in multiple cancers. Dose response curve of FLX in U87, Panc1 and HT29 cells. Data are presented as mean $\pm S E M$ of 3-5 independent experiments performed in quadruplicate. ANOVA, $p<0.001$, followed by t-test where $* * p<0.01, * * * p<0.001$, compared to vehicle-treated control.

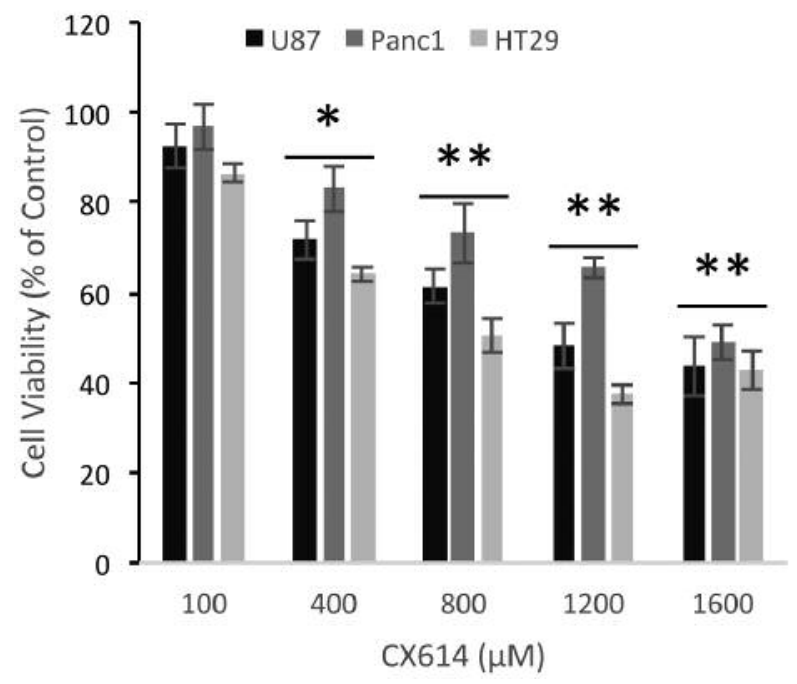

Figure 2. CX614 is oncolytic in multiple cancers. Dose response curve of CX614 in U87, Panc1 and HT29 cells. Data are presented as mean \pm SEM of 3-4 independent experiments performed in quadruplicate. ANOVA, $p<0.01$, followed by t-test where $* p<0.05$, **p<0.01, compared to vehicle-treated control.
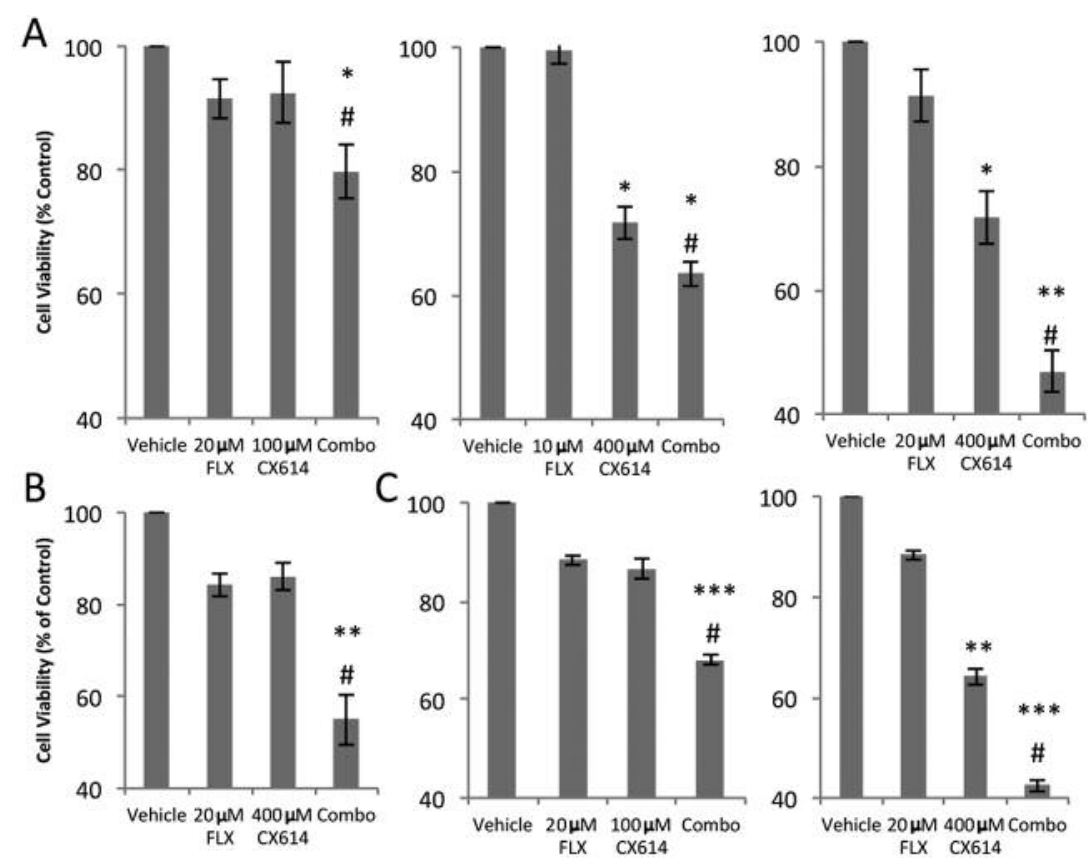

Figure 3. CX614 enhances FLX's oncolytic effects. FLX and CX614 synergistically reduce A) U87, B) Panc1 and C) HT29 cell viability. Data are presented as mean $\pm S E M$ of 3-4 independent experiments performed in quadruplicate. T-test where $* p<0.05$, **p $<0.01$, compared to vehicle-treated control. ${ }^{\#} p<0.05$, king's synergy test, demonstrating significant synergistic interaction.

maturation, differentiation and proliferation, BDNF also exerts similar effects in cancer cells $(15,16)$. Thus, it was somewhat perplexing to observe that class I ampakines inhibit growth of several cancer cell lines in vitro. Furthermore, up regulation of BDNF in the hypothalamus has also been implicated in producing an immune-augmenting anti-tumor response against several cancers (17-19). Since the hypothalamus expresses functional AMPARs (20), it is 
possible that ampakines may be able to produce an anti-tumor response in vivo as well as exert a small, direct oncolytic effect at clinically relevant concentrations.

While a considerable number of studies have suggested that the antidepressant property of FLX was due to its inhibition of serotonin reuptake (21), more recent data suggest that FLX may elicit its anti-depressant effects by activating AMPA receptors. Though its activity as an AMPA receptor agonist was only vigorously demonstrated recently (5), its pre-clinical interactions with AMPAR PAMs were demonstrated over a decade ago (21). The elegant studies performed by $\mathrm{Li}$ et al. (21) demonstrate synergistic antidepressant interactions between ampakines and several SSRIs, including FLX. They also show that tricyclic antidepressants (TCA) and ampakines synergistically reduce depressive behavior in vivo. TCA also have been shown to act as AMPAR agonists at moderate micromolar concentrations (22) lending more credit to the possibility that a broad range of drugs, used until now as antidepressants, might also have clinical utility as oncolytics by augmenting AMPAergic currents and downstream signaling pathways.

It is becoming more widely accepted that tumors are heterogenous in nature and that cells within a tumor exhibit canonical characteristics associated with cancer (increased proliferation, migration and invasion), while a small portion of tumor cells divide infrequently and are much more resistant to DNA damaging agents frequently used to target the tumor (23). This subpopulation is referred to as cancer stem cells and are believed to be the reason many cancers rebound after what is considered successful treatment. Since the first identification of cancer cells positive for certain stem cell markers, considerable efforts have been made to isolate and characterize these cells in order to determine their vulnerabilities. Though progress has been slow, one recent report suggested that cancer stem cells originating from the brain over-express CP-AMPARs, compared to cancer cells that already overexpress CP-AMPARs (23). If this preliminary result proves to be true, it raises the possibility of using drugs that increase AMPAergic activity, both direct agonists as well as ampakines, to target both rapidly dividing cancer cells and quiescent cancer stem cells. With particular respect to FLX, synergistic interactions have already been demonstrated with common chemotherapies (reviewed by (24)).

Taken together, the present data demonstrate that CX614, a Class I ampakine, and FLX individually inhibit cancer cell growth. Effects that are synergistically enhanced when the two drugs are combined. We believe this is accomplished by CX614 increasing FLX affinity for the AMPA receptor resulting in greatly enhanced calcium influx and subsequent signaling pathways. Class II ampakines, that do not increase AMPAR agonist binding affinity, did not inhibit U87 cell viability or synergistically inhibit U87 cell viability when paired with FLX, suggesting that the oncolytic effects of FLX observed in the current work are probably AMPAR-dependent.

\section{References}

1 Staubli U, Rogers G and Lynch G: Facilitation of glutamate receptors enhances memory. PNAS 91(2): 777-781, 1994.

2 Radin D, Zhong S, Purcell R and Lippa A: Acute ampakine treatment ameliorates age-related deficits in long-term potentiation. Biomed and Pharmacother 6(84): 806-809, 2016.

3 Arai AC and Kessler M: Pharmacology of ampakine modulators: From AMPA receptors to synapses and behavior. Current Drug Targets 8(5): 583, 2007.

4 Arai AC, Xia YF, Rogers G, Lynch $G$ and Kessler M: Benzamide-type ampa receptor modulators form two subfamilies with distinct modes of action. J Pharmacol Exp Ther 303(3): 1075-1085, 2002.

5 Liu KH, Yang ST, Lin YK, Lin JW, Lee YH, Wang JY, Hu CJ, Lin EY, Chen SM, Then CK and Shen SC: Fluoxetine, an antidepressant, suppresses glioblastoma by evoking amparmediated calcium-dependent apoptosis. Oncotarget 6(7): 50885101, 2015.

6 Herner A, Sauliunaite D, Michalski CW, Erkan M, De Oliveira T, Abiatari I, Kong B, Esposito I, Friess H and Kleeff J: Glutamate increases pancreatic cancer cell invasion and migration via ampa receptor activation and kras-mapk signaling. Int J Cancer 129(10): 2349-2359, 2011.

7 Ripka S, Riedel J, Neesse A, Griesmann H, Buchholz M, Ellenrieder V, Moeller F, Barth P, Gress TM and Michl P: Glutamate receptor gria3 - target of cux 1 and mediator of tumor progression in pancreatic cancer. Neoplasia 12(8): 659-667, 2010.

8 Stepulak A, Rola R, Polberg K and Ikonomidou C: Glutamate and its receptors in cancer. J Neural Transm (Vienna) 121(8): 933-944, 2014.

9 Radin D, Lippa A, Patel P and Leonardi D: Lifeguard inhibition of fas-mediated apoptosis: A possible mechanism for explaining the cisplatin resistance of triple-negative breast cancer cells. Biomed Pharmacother 77: 161-166, 2016.

10 Kumar V, Radin D and Leonardi D: Probing the oncolytic and chemosensitizing effects of dihydrotanshinone in an in vitro glioblastoma model. Anticancer Res 37: 6025-6030, 2017.

11 Arai AC, Kessler M, Rogers G and Lynch G: Effects of the potent ampakine cx614 on hippocampal and recombinant ampa receptors: Interactions with cyclothiazide and gyki 52466. Mol Pharmacol 58(4): 802-813, 2000.

12 Lee CS, Kim YJ, Jang ER, Kim W and Myung SC: Fluoxetine induces apoptosis in ovarian carcinoma cell line ovcar-3 through reactive oxygen species-dependent activation of nuclear factorkappab. Basic Clin Pharmacol Toxicol 106(6): 446-453, 2010.

13 Schitine C, Xapelli S, Agasse F, Sarda-Arroyo L, Silva AP, De Melo Reis RA, de Mello FG and Malva JO: Ampakine cx546 increases proliferation and neuronal differentiation in subventricular zone stem/progenitor cell cultures. Eur J Neurosci 35(11): 1672-1683, 2012.

14 Lauterborn JC, Lynch G, Vanderklish P, Arai A and Gall CM: Positive modulation of ampa receptors increases neurotrophin expression by hippocampal and cortical neurons. J Neurosci 20(1): 8-21, 2000.

15 Jaboin J, Kim CJ, Kaplan DR and Thiele CJ: Brain-derived neurotrophic factor activation of trkb protects neuroblastoma cells from chemotherapy-induced apoptosis via phosphatidylinositol 3'-kinase pathway. Cancer Res 62(22): 6756-6763, 2002. 
16 Jia S, Wang W, Hu Z, Shan C, Wang L, Wu B, Yang Z, Yang X and Lei D: Bdnf mediated trkb activation contributes to the emt progression and the poor prognosis in human salivary adenoid cystic carcinoma. Oral Oncol 51(1): 64-70, 2015.

17 Cao L, Liu X, Lin EJ, Wang C, Choi EY, Riban V, Lin B and During MJ: Environmental and genetic activation of a brainadipocyte bdnf/leptin axis causes cancer remission and inhibition. Cell 142(1): 52-64, 2010.

18 Xiao R, Bergin SM, Huang W, Slater AM, Liu X, Judd RT, Lin ED, Widstrom KJ, Scoville SD, Yu J, Caligiuri MA and Cao L: Environmental and genetic activation of hypothalamic bdnf modulates t-cell immunity to exert an anticancer phenotype. Cancer Immunol Res 4(6): 488-497, 2016.

19 Radin DP and Patel P: Bdnf: An oncogene or tumor suppressor? Anticancer Res 37(8): 3983-3990, 2017.

20 Ferreira-Neto HC, Antunes VR and Stern JE: Atp stimulates rat hypothalamic sympathetic neurons by enhancing ampa receptormediated currents. J Neurophysiol 114(1): 159-169, 2015.

$21 \mathrm{Li}$ X, Witkin JM, Need AB and Skolnick P: Enhancement of antidepressant potency by a potentiator of ampa receptors. Cell Mol Neurobiol 23(3): 419-430, 2003.
22 Stoll L, Seguin S and Gentile L: Tricyclic antidepressants, but not the selective serotonin reuptake inhibitor fluoxetine, bind to the s1s2 domain of ampa receptors. Arch Biochem Biophys 458(2): 213-219, 2007.

23 Oh MC, Kim JM, Safaee M, Kaur G, Sun MZ, Kaur R, Celli A, Mauro TM and Parsa AT: Overexpression of calcium-permeable glutamate receptors in glioblastoma derived brain tumor initiating cells. PLoS One 7(10): e47846, 2012.

24 Radin DP and Patel P: A current perspective on the oncopreventive and oncolytic properties of selective serotonin reuptake inhibitors. Biomed Pharmacother 87: 636-639, 2017.

Received October 28, 2017

Revised November 6, 2017

Accepted November 7, 2017 